This document is confidential and is proprietary to the American Chemical Society and its authors. Do not copy or disclose without written permission. If you have received this item in error, notify the sender and delete all copies.

\title{
Chemical Vapor Deposition of High Quality Graphene Films from Carbon Dioxide Atmospheres
}

\begin{tabular}{|r|l|}
\hline \multicolumn{1}{|r|}{ Journal: } & ACS Nano \\
\hline Manuscript ID: & nn-2014-04822m.R2 \\
\hline Manuscript Type: & Article \\
\hline Date Submitted by the Author: & $12-$ Nov-2014 \\
\hline Complete List of Authors: & $\begin{array}{l}\text { Strudwick, Andrew; BASF SE, } \\
\text { Weber, Nils; BASF SE, } \\
\text { Schwab, Matthias; BASF SE, } \\
\text { Kettner, Michael; BASF SE, } \\
\text { Weitz, R. Thomas; BASF SE, Organic Electronics } \\
\text { Wünsch, Josef; BASF SE, } \\
\text { Müllen, Klaus; Max-Planck-Institute for Polymer Research, } \\
\text { Sachdev, Hermann; Max-Planck-Institute for Polymer Research, }\end{array}$ \\
\hline
\end{tabular}

\section{SCHOLARONE ${ }^{m}$ \\ Manuscripts}




\title{
Chemical Vapor Deposition of High Quality
}

\section{Graphene Films from Carbon Dioxide Atmospheres}

\author{
Andrew James Strudwick ${ }^{1 \dagger}$, Nils Eike Weber ${ }^{1}$, Matthias Georg Schwab ${ }^{1}$, Michel Kettner ${ }^{2,3}$, R. \\ Thomas Weitz ${ }^{2,3}$, Josef R. Wünsch ${ }^{1}$, Klaus Müllen ${ }^{1,4^{*}}$, Hermann Sachdev ${ }^{1,4^{*}}$ \\ 1 BASF SE, Carbon Materials Innovation Center, 67056, Ludwigshafen, Germany. \\ 2 BASF SE, OFET Systems, 67056 Ludwigshafen, Germany. \\ 3 Innovation Lab GmbH, Speyerer Straße 4, 69115 Heidelberg, Germany. \\ 4 Max-Planck-Institute for Polymer Research (MPI-P), Ackermannweg 10, 55128 Mainz, \\ Germany.
}

\section{Corresponding Authors}

sachdev@,mpip-mainz.mpg.de; muellen@mpip-mainz.mpg.de;

$\dagger$ now at: 2-DTech Limited, Core Technology Facility, 46 Grafton Street, Manchester, M13

9NT, UK

Keywords: graphene, chemical vapor deposition, Raman microscopy, charge carrier mobility, scanning electron microscope. 


\section{ToC Figure}

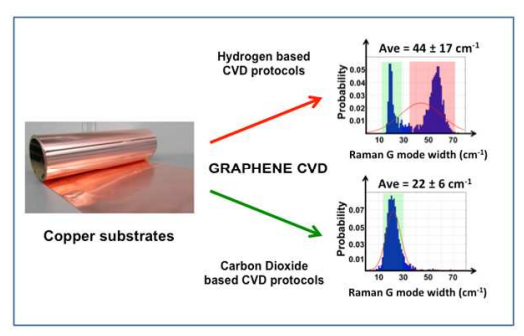

\section{$\underline{\text { Abstract }}$}

The realization of graphene-based, next-generation electronic applications essentially depends on a reproducible, large-scale production of graphene films via chemical vapor deposition (CVD). We demonstrate how key challenges such as uniformity and homogeneity of the copper metal substrate as well as the growth chemistry can be improved by the use of carbon dioxide and carbon dioxide enriched gas atmospheres. Our approach enables graphene film production protocols free of elemental hydrogen and provides graphene layers of superior quality compared to samples produced by conventional hydrogen/methane based CVD processes. The substrates and resulting graphene films were characterized by scanning electron microscopy (SEM), energy dispersive X-ray spectroscopy (EDX) and Raman microscopy, sheet resistance and transport measurements. The superior quality of the as-grown graphene films on copper is indicated by Raman maps revealing average $\mathrm{G}$ band widths as low as $18 \pm 8 \mathrm{~cm}^{-1}$ at $514.5 \mathrm{~nm}$ excitation. In addition, high charge carrier mobility's of up to $1975 \mathrm{~cm}^{2} / \mathrm{Vs}$ were observed for electrons in transferred films obtained from a carbon dioxide based growth protocol. The enhanced graphene film quality can be explained by the mild oxidation properties of carbon dioxide, which at high temperatures enables an uniform conditioning of the substrates by an efficient removal of preexisting and emerging carbon impurities and a continuous suppression and in-situ etching of carbon of lesser quality being co- deposited during the CVD growth. 
The production of high quality graphene films by chemical vapor deposition (CVD) has demonstrated great potential to supply the increasing demand of this material for the realization of next-generation electronics applications. ${ }^{1,2}$ These graphene films ideally consist of either a single layer or few layers of a two- dimensional, hexagonal array of tri-coordinate carbon atoms. The utilization of the outstanding properties of graphene is being screened for a huge variety of applications, such as transistors, ${ }^{3-5}$ sensors $^{6}{ }^{6}$ actuators ${ }^{7}$ and flexible and transparent conducting layers. ${ }^{8}$ From 2008 onwards, ${ }^{9-11}$ the CVD of graphene films on metal substrates like copper ${ }^{11,12}$ and nickel, ${ }^{3,10,13}$ along with the development of transfer techniques onto target substrates, ${ }^{14-16}$ has advanced significantly and is considered for the scaling-up of these methods. ${ }^{1}$ However, there are still important issues to be addressed for a consistent and scalable production of large area high quality graphene films, ideally being of single crystalline nature. ${ }^{17-21}$ These include the uniformity and homogeneity of the substrates, a growth chemistry preferably free of elemental hydrogen and favorable for industrial CVD protocols, i.e. in a pressure range of $\sim 10^{-3}$ mbar to ambient conditions. ${ }^{22,23}$ Also, substrate and growth specific effects, such as e.g. morphological faults, variations in the nucleation density and grain boundaries within the film influence and contribute to the limitations of the sheet resistances and charge carrier mobilities. These intrinsic factors of the graphene layer need to be controlled in the growth process itself before a films transfer onto dielectric substrates for further electrical characterization. The latter step can be associated with the introduction of further defects and unintentional doping, too. For the CVD growth of graphene films, especially the reliable removal of undesired and ubiquitous carbon from the starting substrate, the prevention of uncontrolled graphene nucleation as well as the suppression of low quality carbon co-deposition are important challenges for an industrially 
relevant CVD process. To date, reports on the graphene film CVD on copper substrates still vary with respect to the observed defects and reveal inconsistencies in the as-grown and processed layers, which is e.g. evidenced by the Raman spectra, variations in sheet resistances and electrical transport properties. One important parameter for the graphene growth by CVD is the quality of the copper substrates. Effects related to the presence of copper oxides on the substrates were recently discussed by Hao et al. ${ }^{24}$ to have a significant influence on the graphene nucleation mechanism. ${ }^{25,26}$ The issue of carbon contaminants and its removal by plasma assisted methods was discussed by Kato et al. ${ }^{27}$. However, such continuous plasma processes involving atomic hydrogen can alter the graphene structure itself and induce further structural changes and defects. $^{28,29}$ Carbon contaminations of undefined origin on copper substrates are a general problem for a consistent and reproducible graphene film growth, especially since the metal purity is mostly specified on the metal basis only. Currently, a thermal treatment of the metal substrates in a hydrogen atmosphere prior to the growth stage is described in most scientific reports on CVD graphene for the conditioning of the substrate to achieve a defined surface state. ${ }^{10}$ The chemical nature of hydrogen is believed to be sufficient to reduce and thereby remove carbon residues as well as native oxides. ${ }^{25}$ However, we present evidence to the contrary and describe how such carbonaceous material can lead to an uncontrolled nucleation and growth of poor quality carbon-rich films prior to the actual graphene CVD growth. Therefore, a gas phase etchant for carbon is needed, which eliminates any ubiquitous and undesired carbon sources from the substrates in situ, prevents uncontrolled graphene nucleation and growth and removes codeposited carbon of lesser structural quality than graphene in a mere thermal CVD process. We consider such a method, in combination with a growth process, extremely valuable for advancing the CVD synthesis of graphene films. 
In this report, we i) classify the behavior and batch dependence of copper substrates commonly used for the production of graphene films by surface characterization techniques with respect to the origin and nature of adventitious carbon present or formed during thermal treatment and its removal by hydrogen, ii) use carbon dioxide as a mild oxidant and chemical reactant for the elimination of such unwanted carbon, iii) further develop this carbon dioxide based etching procedure into a continuous CVD growth chemistry for graphene films and iv) characterize the electronic properties of selected films in field effect transistor (FET) devices to demonstrate the high electronic quality of graphene grown by from the newly developed growth protocols.

\section{$\underline{\text { Results and Discussion }}$}

For the mass production of large area coatings of graphene films by CVD, economic copper substrates of adequate quality are required. Therefore, different standard grade commercial copper foils commonly used for graphene CVD were screened in this study. Three different batches of comparable quality, labeled as B1, B2 and B3, were examined with further details given in the experimental section. The substrate pre-treatment and CVD growth presented here was performed in gas atmospheres as subsequently described at $1060^{\circ} \mathrm{C}$ to enable a maximum thermal activation without melting the copper substrates. The response of these copper substrates to the applied procedures and growth conditions under low-pressure thermal CVD was characterized in a step-wise fashion to enable a comparison with the results of graphene CVD growth from commonly applied hydrogen/methane gas atmospheres.

To better understand the individual effects, we focused on five topics, i) how intrinsic sources of carbon originating from the copper foils act upon hydrogen and carbon dioxide pre-treatment 
for carbon removal ( $c f$. Figure 1a-d); ii) how a hydrogen and a carbon dioxide based cleaning step prior to a conventional hydrogen/methane based graphene CVD growth process influences the film quality ( $c f$. Figure 1e-h); iii) how hydrogen as well as carbon dioxide act upon and etch a pre-existing graphene film ( $c f$. Figure 2); iv) how a combination of the pretreatment and continuous in-situ etching with carbon dioxide enables the formation of higher quality graphene films from methane ( $c f$. Figure 4), and v) characterize the electronic properties of selected films (cf. Figure 5).

Raman spectroscopy ${ }^{29-31}$ is a key metrology method for graphene characterization. It allows to determine its structural perfection by analyzing the spectroscopic features of the $D, G$ and $2 \mathrm{D}$ modes (like their presence, line width and intensities) and can be used as an efficient tool to correlate the quality of the as-grown graphene films to the influence of the CVD parameters. Post-growth transfer steps of the graphene films from the growth substrate onto other target substrates can induce additional doping and defects and thus do inhibit a straightforward correlation of the Raman spectroscopic features of the transferred films with the CVD parameters. Therefore, we have collected representative overview Raman spectra from the asgrown films on the copper substrates ( $c f$. SI) and performed Raman mappings of the G- mode of these CVD graphene films. This Raman mode can be well detected directly on the substrate by excitation with conveniently available laser excitation (e.g. $514.5 \mathrm{~nm})$ and the local information gained by mapping of the samples together with the G-band line width distribution can be used as a characteristic feature for a rapid screening for the presence of carbon and the structural perfection of the CVD graphene films.

Since intrinsic sources of carbon present in the metal substrate will strongly influence the nature of the graphene films produced by CVD, we therefore analyzed copper foils prior to any surface 
and heat treatment via energy dispersive X-ray spectroscopy (EDX) and Raman microscopy. The Raman spectra taken at selected locations of B1 and B2 both contain broad D and G bands with the $2 \mathrm{D}$ mode being absent and thus indicate the carbon present is amorphous. ${ }^{29-31}$ These carbonlike Raman signatures occur more frequently on the surface of B1 copper (Figure SI 1a) when compared to B2 copper (Figure SI 1b), where only isolated carbon domains of micrometer size are detected. The observed carbon peaks in the EDX spectra exemplarily taken from representative copper substrates (Figure SI 1c) further indicate carbon to be a ubiquitous preexisting impurity and present in the bulk of even high quality copper grades. ${ }^{32}$

When B1 and B2 copper substrates were heated prior to graphene growth in a hydrogen atmosphere typically used for cleaning ( $c f$. Fig. 1a,b: $150 \mathrm{sccm}$ hydrogen flow for the whole process, $60 \mathrm{~min}$ at $1060^{\circ} \mathrm{C}$ ), this pre-existing carbon source is sufficient enough to form a carbon-rich surface layer for certain batches of copper like e.g. B1. According to the SEM image in Figure 1a and Raman microscopy, the resulting surface consists of graphitic island domains interspersed between areas of disordered carbon, which actually is blocking access of suitable growth species to the underlying metal substrate. The distribution curve of the Raman G band widths (DRGW; for calculation see SI) on this sample reveals two distinct maxima, which are shaded in green and red for clarity (Figure 1a, inset). The green area of the DRGW corresponds to regions exhibiting graphite crystal like spectra and the red area to disordered carbon like spectra ( $c f$. Figure SI 2; B1-H2-Crystal, B1-H2-Disorder). The green section thus results from surface regions with graphitic structures leading to a distinct Raman signature with a pronounced and narrow G band (DRGW approx. $20.2+/-2.0 \mathrm{~cm}^{-1}$ ) and a multicomponent $2 \mathrm{D}$ mode indicating multiple graphene layers. On the contrary, Raman data collected in between these graphitic domains reveal broad D and G bands typical for a non-crystalline carbon material (red 
section of the DRGW plot). The source of the carbon from which the graphite-like islands are formed appears to be intrinsic to B1 copper, since such islands with similar DRGW's can be observed by a mere heating of the substrate, too. Comparative samples of B2 and B3 copper were produced during the same hydrogen pretreatment as for B1 (thus all three substrates underwent fully identical conditions). In contrast to B1, SEM and Raman analysis of B2 and B3 indicates that both samples do not display any crystalline carbon features on the surface (Figure $1 \mathrm{~b}$ and Figure SI 3a). These result along with regularly observed carbon-like Raman signals in B1 compared to B2 copper ( $c f$. Figure SI 1a,b) correlates to this unwanted carbon film formation from ubiquitous carbon sources and indicates the drawbacks of a mere hydrogen pre-treatment commonly used prior to the growth of graphene films.

However, annealing copper foils from different batches in a mere carbon dioxide atmosphere for the same time and temperature ( $c f$. Fig. 1c,d: $50 \mathrm{sccm}$ carbon dioxide flow for the whole process, $60 \mathrm{~min}$ at $1060^{\circ} \mathrm{C}$ ) removes this carbon film from $\mathrm{B} 1$ copper and makes the surface suitable for graphene growth. We consider this as a significant finding for the development of batch-independent growth protocols for high quality graphene films to reduce defects arising from uncontrolled carbon formation. An SEM analysis of the surface morphology reveals stepped features as shown in Figure 1c and a similar morphology and surface structures are observed for B2 copper (Figure 1d) and B3 ( $c f$. Figure SI 3b) treated for the same conditions with carbon dioxide.

Based on the previous discussed observations, the influence of either a hydrogen or a carbon dioxide pre-treatment followed directly by a graphene film growth from a hydrogen/methane mixture is now being compared (pre-treatment: $60 \mathrm{~min}$ at $1060^{\circ} \mathrm{C}$ with either $150 \mathrm{sccm}$ hydrogen or $50 \mathrm{sccm}$ carbon dioxide; growth for $60 \mathrm{~min}$ from $150 \mathrm{sccm}$ hydrogen / $50 \mathrm{sccm}$ methane). In 
the case of a hydrogen pretreatment, the island-like graphene domains remain for B1 in such a growth process, as indicated by the sample morphology in the SEM (cf. Figure 1e), while the DRGWs are highlighting the quality of the resulting film with the distribution showing two peaks similar as in Figure 1a. For a substrate of B2 copper subjected to the same treatment and growth conditions, Figure 1f reveals that the island-like growth is not present and the DRGW exhibits a single peak in the crystalline graphene region and indicates the formation of a homogeneous graphene film. This striking inconsistency in the graphene quality obtained from two batches of copper seemingly very similar in chemical composition after undergoing the same growth protocol is undesirable for any process carried out for scientific research or on industrial scale. It is evident, that an uncontrolled build-up of a carbon surface layer formed during the pretreatment step ( $c f$. Fig 1a, B1) will subsequently hamper the access of any gas phase growth species to the underlying metal substrate.

We believe that the problematic presence of carbon either in the bulk or on the surface can be ascribed to contaminations from the metal substrate production process and organic residues, e.g. originating from protecting agents. These materials will only partially be affected by wetchemical solvent-based pre-cleaning steps. As demonstrated, these impurities give rise to the build-up of uncontrolled carbon layers by generating volatile growth species in a hydrogen atmosphere. It is to be noted, that such carbon sources are generally believed to be removed by the hydrogen pre-treatment commonly applied in the state of the art of CVD graphene production. However, we have proven by the results from the CVD synthesis of graphene on the pre-treated copper samples as described above this to be not the case.

Therefore, the removal of such carbon sources is imperative for the conditioning and consistent production of high quality graphene on these substrates. It was explored if a mere carbon dioxide 
pre-treatment $\left(60 \mathrm{~min}, 50 \mathrm{sccm}, 1060^{\circ} \mathrm{C}\right)$ prior to the growth step $(60 \mathrm{~min}, 150 \mathrm{sccm}$ hydrogen $/ 50 \mathrm{sccm}$ methane, $1060^{\circ} \mathrm{C}$ ) can serve this purpose. The Figures $1 \mathrm{~g}$ and $1 \mathrm{~h}$ depict the morphologies of graphene films grown on B1 and B2 copper obtained from this procedure. The SEM images reveal now a consistent behavior for both batches, while the island-like patches as previously observed for the case of B1 copper observed by hydrogen pre-treatment are absent. Also, the DRGWs exhibit a single, symmetrical peak with a similar average peak position for both samples of $22 \pm 6 \mathrm{~cm}^{-1}$ (B1) and $20 \pm 5 \mathrm{~cm}^{-1}$ (B2), respectively. In particular, these two values are lower than the $25 \pm 9 \mathrm{~cm}^{-1}$ derived for graphene growth on B2 copper after hydrogen pre-treatment only ( $c f$. Figure 1f, inset), indicating an improved graphene quality also in this case (cf. exemplary Raman spectra in Figure SI2). 
a)

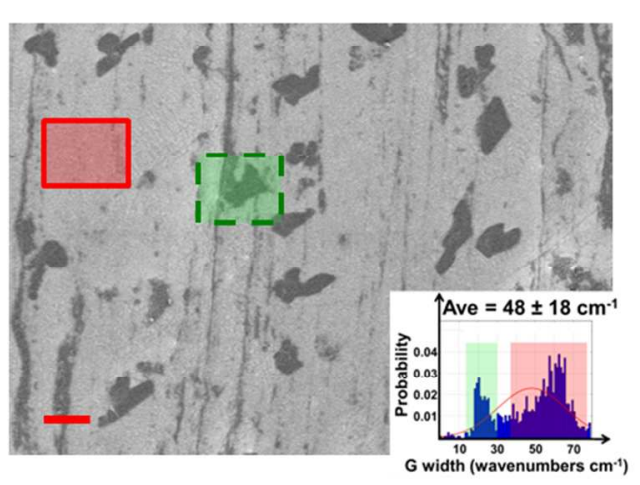

Batch 2

Batch 1 hydrogen pre-treatment without subsequent CVD graphene growth

b)

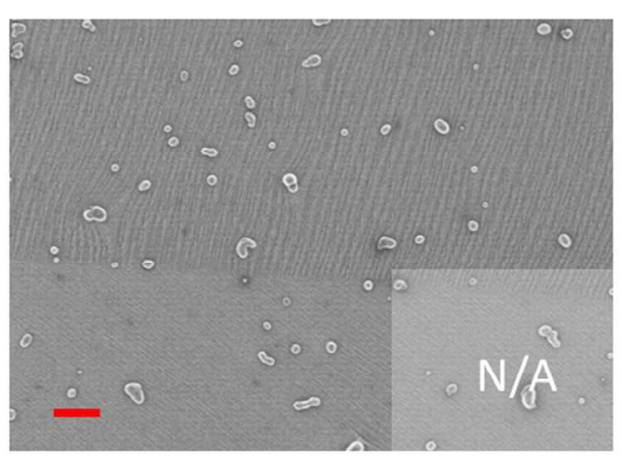

carbon dioxide pre-treatment without subsequent CVD graphene growth

C)

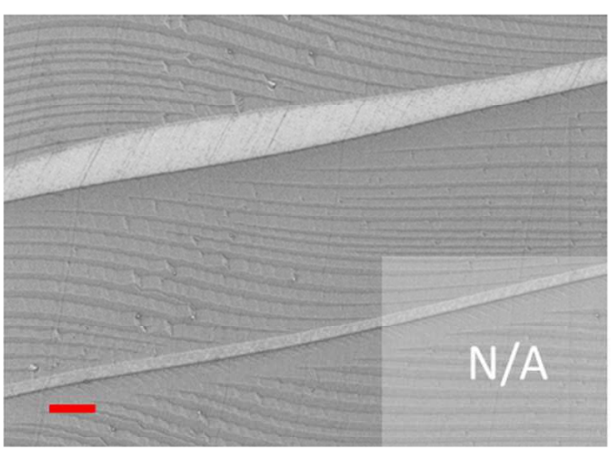

d)

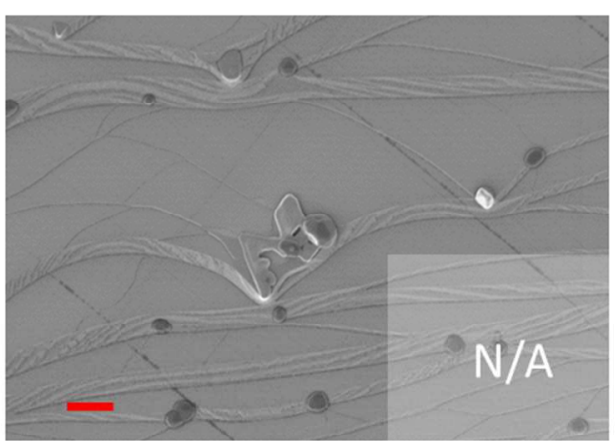

hydrogen pre-treatment with subsequent CVD graphene growth

e)

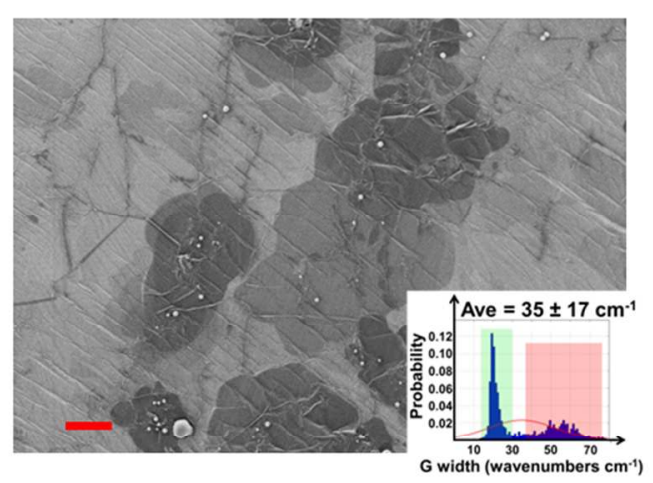

f)

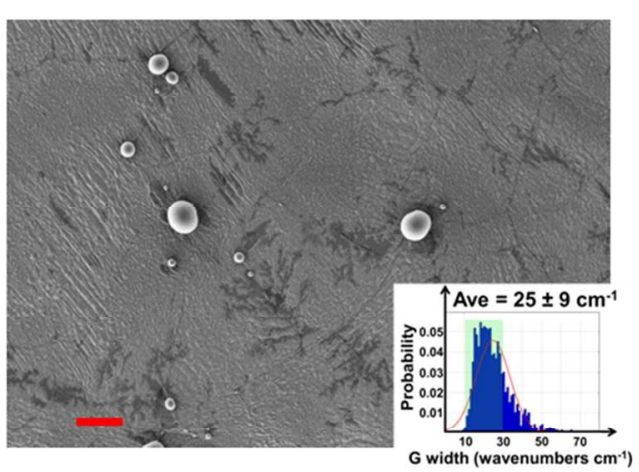

carbon dioxide pre-treatment with subsequent CVD graphene growth

g)



h)

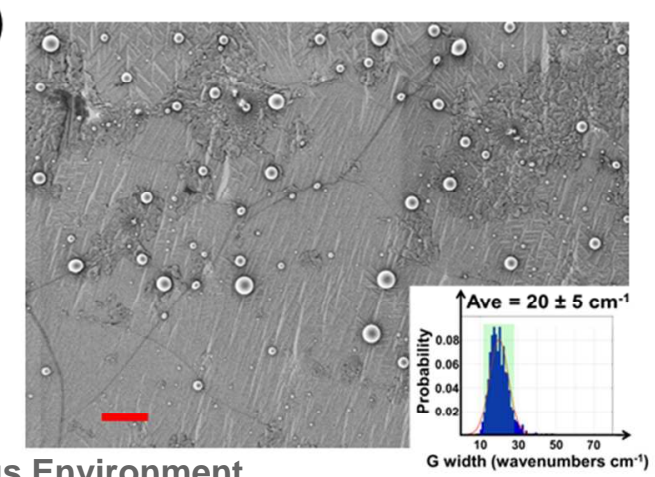


Figure 1. SEM pictures and distribution of Raman G band widths (DRGWs, $c f$. insets) for B1 and B2 copper substrates having undergone different treatments as quoted in the figures at a temperature of $1060{ }^{\circ} \mathrm{C}$. Gas flows (where applicable) for hydrogen pre-treatment $150 \mathrm{sccm}$; for carbon dioxide pre-treatment $50 \mathrm{sccm}$; for graphene growth $150 \mathrm{sccm}$ hydrogen / $50 \mathrm{sccm}$ methane. In the DRGWs, the green region highlights signals resulting from crystalline graphene, and the red region those from disordered carbon. The position of such signals with respect to the features observed in SEM images is highlighted by matching colored boxes in Fig. 1a. Red scale bars indicate 1 $\mu \mathrm{m}$. Further details of a typical heating protocol are given in SI Figure 5.

For a proof of concept, the influence of hydrogen and carbon dioxide on pre-existing CVD graphene was additionally characterized. For this purpose, a graphene film grown under standard conditions on $\mathrm{B} 1$ copper (pre-treatment:150 sccm hydrogen flow, $60 \mathrm{~min}, 1060^{\circ} \mathrm{C}$; graphene growth: $150 \mathrm{sccm}$ hydrogen/50sccm methane, $270 \mathrm{~min}, 1060^{\circ} \mathrm{C}$ ) was first analyzed by SEM and Raman microscopy and then subjected to either a hydrogen or a carbon dioxide atmosphere at high temperature (Figure 2). The SEM and DRGW data indicate for this graphene film on copper (referred to as 'Sample $\mathrm{X}^{\prime}, c f$. Figure 2a) an island-like morphology of graphitic carbon to be present, too, which persists even up to these long growth times. The DRGW exhibits a similar bimodal peak distribution as observed for this type of copper substrate for shorter growth times (cf. Figure 1e). After this characterization, 'Sample X was split into two sections. One section was subjected to a post-treatment in a mere hydrogen atmosphere (60 minutes, $150 \mathrm{sccm}$ flow, $1060^{\circ} \mathrm{C}$; labeled 'Sample $\mathrm{X}+\mathrm{H}_{2}$ '), while the other section was treated in a carbon dioxide atmosphere (60 minutes, $50 \mathrm{sccm}, 1060^{\circ} \mathrm{C}$; labeled `Sample $\left.\mathrm{X}+\mathrm{CO}_{2}\right)$.

The morphology of the sample obtained from the post-processing in a hydrogen atmosphere (cf. Figure 2b; 'Sample $\mathrm{X}+\mathrm{H}_{2}$ ') exhibits a similar island-like film structure as observed for the as-grown graphene film ( $c f$. Figure 2a; 'Sample $\mathrm{X}$ ') with a comparable characteristic bimodal 
a)

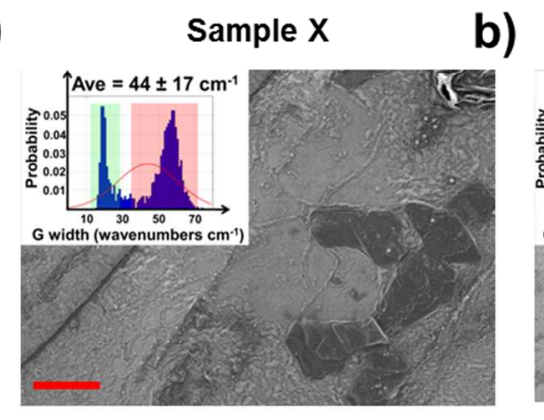

DRGW with slightly reduced contributions of modes at higher half widths. This clearly constitutes further proof for hydrogen not to be sufficiently suitable to remove graphene, graphitic carbon and carbonaceous deposits under the applied thermal conditions. On the contrary, the applied carbon dioxide treatment fully removes the graphene film and the SEM data (cf. Figure 2c;'Sample $\mathrm{X}+\mathrm{CO}_{2}{ }^{\prime}$ ) reveal a clean copper surface of a step-like morphology without any carbon detectable by Raman spectroscopy. Selected Raman spectra were extracted from the mapping and proof the absence of any carbon on this sample (cf. SI Figure 3c). These findings unambiguously highlight the reproducible ability of carbon dioxide to etch off carbon form the copper substrates in a gas phase reaction better than hydrogen.

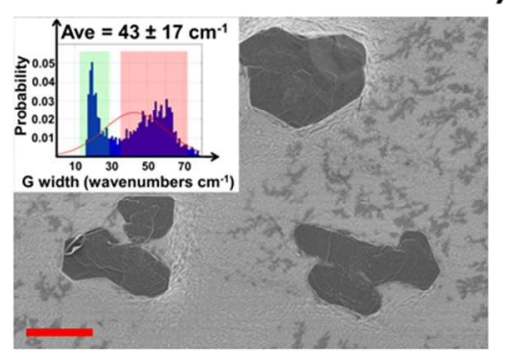

c)

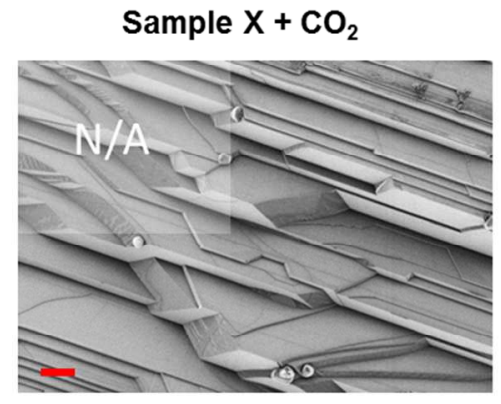

Figure 2. For all samples a distribution of Raman G band widths (DRGWs) is given as an inset wherever applicable. Red scale bar indicates $1 \mu \mathrm{m}$. (a) SEM image from 'Sample X' revealing island-like graphene growth (B1 copper substrate, pre-treated for $60 \mathrm{~min}$. in $150 \mathrm{sccm}$ hydrogen flow followed by graphene growth for $270 \mathrm{~min}$ from a mix of $50 \mathrm{sccm}$ methane/150 sccm hydrogen at $1060{ }^{\circ} \mathrm{C}$ ); (b) SEM image from 'Sample $\mathrm{X}+\mathrm{H}_{2}$ ' which still exhibits island-like graphene on the surface despite an extra heat treatment with $150 \mathrm{sccm}$ hydrogen flow at $1060^{\circ} \mathrm{C}$ for 60 min.; (c) SEM image from 'Sample $\mathrm{X}+\mathrm{CO}_{2}$ ' from a heat treatment with 50 sccm carbon dioxide for 60 min at $1060^{\circ} \mathrm{C}$. The graphene and surplus carbon were fully removed and no Raman signals were detectable (cf. SI Fig. 3c).

We also verified the effect of pre-treating the substrates in mixed atmospheres composed of hydrogen and carbon dioxide to investigate possible synergistic effects. 
In Figure 3, the bar chart summarizes the Raman mapping data for a statistical evaluation of the G mode of graphene films grown on B1, B2 and B3 copper substrates in-situ directly after high temperature pre-cleaning procedures in a hydrogen atmosphere, in a hydrogen/carbon dioxide mixture and in a carbon dioxide atmosphere ( $c f$. representative Raman spectra for each procedure in Figure SI 2). The average Raman G band width decreases for graphene films on all copper batches when carbon dioxide is introduced into the pre-treatment step (either in a mixed or pure state) and indicates an enhancement of the graphene film quality. The most significant improvement is observed in the Raman spectrum and width of the $G$ band for a film grown on B1 copper, which becomes completely graphene-like once the growth-limiting carbon film is removed by carbon dioxide in the substrate pre-treatment stage. However, the post-growth analysis indicated that carbon dioxide is the main driving force for the improved surface cleaning since the quality of the graphene samples synthesized on such copper foils remains virtually unaffected as compared to pure carbon dioxide annealing.

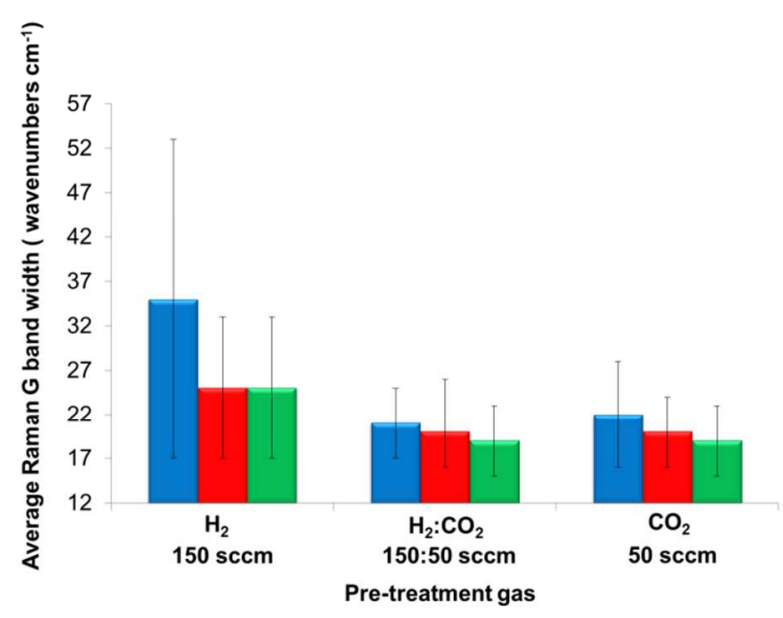

Figure 3. Bar charts of average Raman $G$ band widths extracted from the corresponding DRGW for graphene films produced on B1 copper (blue), B2 copper (red) and B3 copper (green). Samples were obtained from gas pre- 
treatments for $60 \mathrm{~min}$ at $1060^{\circ} \mathrm{C}$ as specified before subsequent in-situ graphene growth from $50 / 150 \mathrm{sccm} \mathrm{CH} / \mathrm{H}_{2}$ for 60 minutes at the same temperature.

With the experiments reported above, we could demonstrate that organic residues, which are present on the surface or as inclusions in the bulk and may originate from the foil production process, together with other sources of ubiquitous carbon can lead to the formation of morphological changes on the surface during the high temperature process and, more importantly, give rise to the build-up of pyrolytic non-graphitic carbon films by generating volatile growth species in a hydrogen containing atmosphere. Such carbon sources are generally supposed to be removed by the hydrogen pre- treatment, which apparently is not the case even by extending the reaction times $(c f$. Figure $2 \mathrm{a}, \mathrm{b})$. With our experiments we further confirmed the oxidizing nature of carbon dioxide towards carbon and carbonaceous matter and its improved ability to efficiently remove such matter in comparison to the conventionally used thermal hydrogen pre-treatment. The copper substrates still retain their metallic character on the surface by the high temperature pre-treatment in a carbon dioxide atmosphere, since directly after such an exposure no traces of copper oxides were detectable by Raman spectroscopy (cf. SI Figure 3c). Carbon, e.g. on the substrate surface or expelled from the bulk, can react even during thermally activated processes, with carbon dioxide and is being converted into carbon monoxide, a reaction described as the Boudouard equilibrium and of importance for CVD. ${ }^{33-35}$ This reaction ( $\mathrm{C}+\mathrm{CO}_{2} \leftrightarrow 2 \mathrm{CO}$ ) is a textbook example for a reversible chemical equilibrium of general importance in chemistry and describes the reaction of solid carbon with carbon dioxide to form carbon monoxide (or equally implying carbon can be formed in a reverse reaction by disproportionation of carbon monoxide). The reaction pathway (dissolution of carbon by carbon 
dioxide or carbon formation from carbon monoxide) is temperature- and pressure dependent and here we used an excess of carbon dioxide at high temperatures to remove traces of carbon by applying this chemical equilibrium. Thus, the use of this chemistry leads to a removal of undesirable pre-existing carbon from the substrates as well as carbon formed during the initial stage prior to the actual growth step. The data from the previously discussed set of experiments indicate that the removal proceeds faster with defective and less structured carbon due to kinetic effects. In addition, carbon rich solids (generalized as $\mathrm{C}_{\mathrm{x}} \mathrm{H}_{\mathrm{y}} \mathrm{O}_{\mathrm{z}}$ ) may be formed during the heat treatment form organic residues, which react with carbon dioxide in a similar manner, but with the formation of further byproducts like water and volatile oxygen containing hydrocarbon species. Small features occasionally occurring in SEM on the film surface, e.g. in Figures 1 and 2, are comparable to those observed by others (e.g. Kim et al. $\left.{ }^{36}\right)$.

In the previous section of our discussion we demonstrated how carbon and carbonaceous sources being present in copper foils used for graphene CVD strongly influence the quality of the resulting films. Especially, the uncontrollable formation of carbon of inferior quality to graphene, either being deposited from the gas phase or being extruded from the bulk copper hinders the formation of a complete layer of high quality graphene. Such a carbon formation is fully suppressed in a carbon dioxide atmosphere in contrast to a hydrogen pre-treatment.

\begin{abstract}
After investigation of the role of carbon dioxide during the first stages of the CVD graphene process we further extended our experiments to the actual growth stage. Hydrogen not only seems to have a limited effect during the thermal annealing procedure but is in general considered to be detrimental to the speed and quality of graphene production on copper substrates, since it enables the formation of $\mathrm{sp}^{3}$ - bound carbon species, too. ${ }^{37}$ Furthermore, the
\end{abstract}


use of hydrogen is associated with an additional risk potential, which needs to be considered for the industrial scaling of graphene film production with CVD techniques. We consequently considered implementing the beneficial properties of carbon dioxide into a hydrogen-free growth protocol.

So far, there are few reports on hydrogen free CVD protocols for the controlled growth of nondoped graphene ${ }^{38}$ under non-UHV conditions, such as e.g. a rapid thermal CVD with methane as a precursor ${ }^{8}$ or a carbon dioxide - acetylene based approach to improve the graphene quality. ${ }^{39}$ However, commercial acetylene is a source of other C-H-O species, notably acetone. This agent is used as a storage stabilizer and can act as a CVD precursor for graphene growth by itself 40 besides further uncontrolled reactions with the pre-set CVD gas atmosphere at high temperatures. To avoid any unwanted side effects, we focused on a CVD process with fully controlled precursors and parameters by using carbon dioxide and methane as the only reactants for the synthesis of the graphene film. We performed three different experiments to elucidate the potential of a carbon dioxide based graphene growth [i) hydrogen pretreatment - methane only growth; ii) carbon dioxide pretreatment - methane only growth; and iii) carbon dioxide pretreatment - methane/carbon dioxide combined growth]. 
Heat treatment

a) Temperature

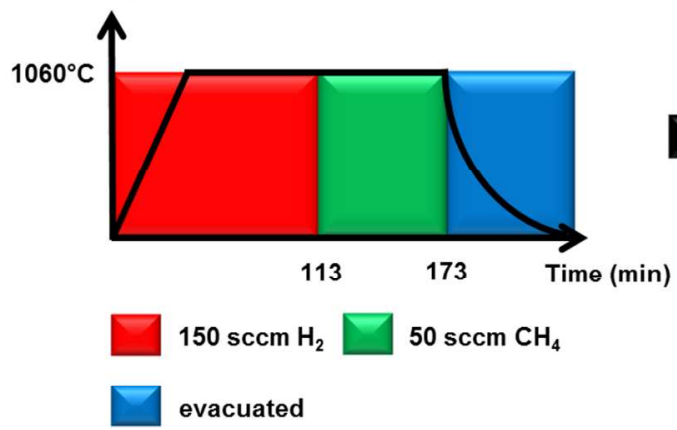

c)

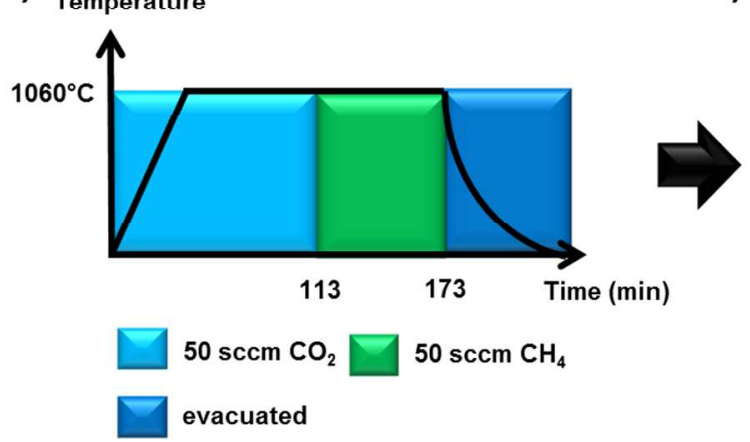

e)

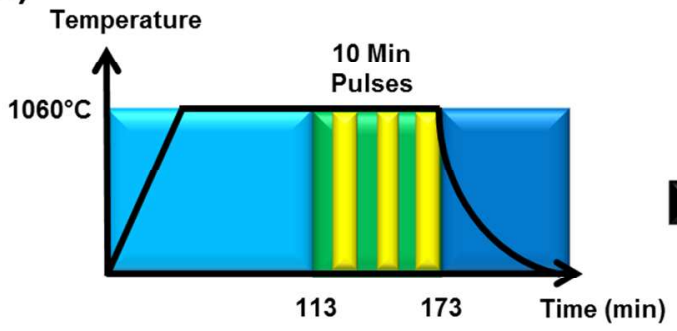

$50 \mathrm{sccm} \mathrm{CO}{ }_{2} \square 0 \mathrm{sccm} \mathrm{CH}_{4}$

\begin{tabular}{l|l} 
evacuated & $25: 50 \mathrm{sccm} \mathrm{CO}: \mathrm{CH}_{4}$
\end{tabular}

f) b)

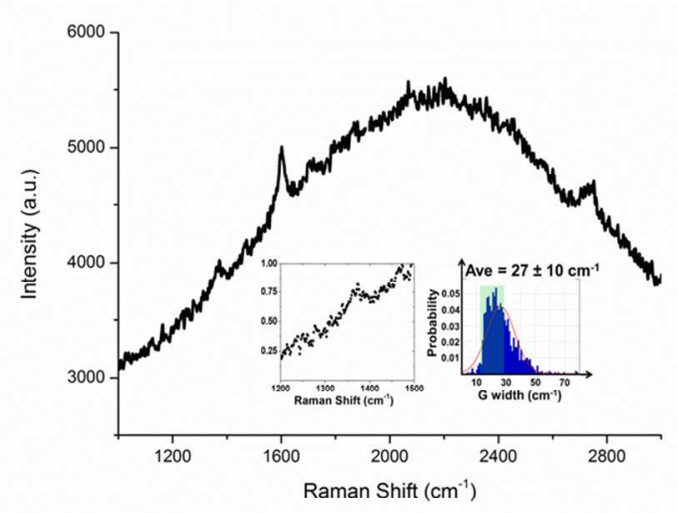

d)
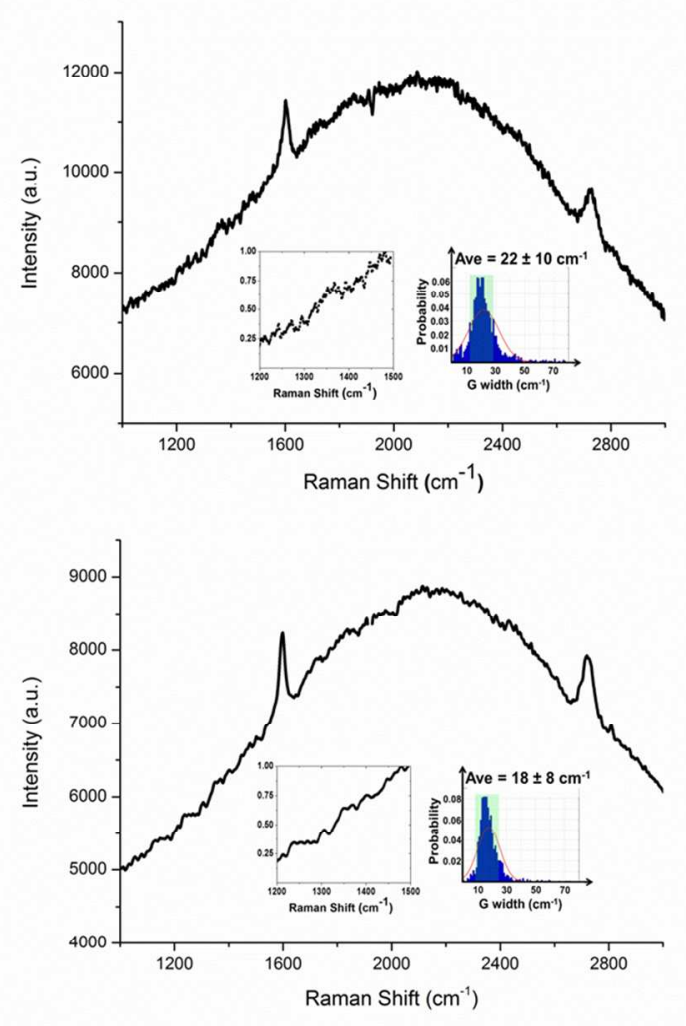

Figure 4. (a) (c) (e) Heating protocols summarizing different annealing conditions followed by hydrogen-free graphene growth procedures on B2 copper. (b) (d) (f) Single representative Raman spectra from each experiment. Left inset: normalized Raman D band spectrum from the main spectrum. Right inset: DRGW plots extracted from Raman maps taken on each sample, respectively. 
The results obtained for these set of experiments are summarized in Figure 4 for B2 copper substrates and in SI Figure 4 for B3 substrates. Figure 4a displays the heating protocol for a control experiment of a copper substrate (B2) with a hydrogen pre-treatment followed by growth with neat methane at $1060^{\circ} \mathrm{C}$. The sample from this protocol exhibits a characteristic graphenelike Raman spectrum ( $c f$. Figure $4 \mathrm{~b}$ ). The inset of Figure $4 \mathrm{~b}$ reveals however the presence of defects indicated by the Raman D band and the DRGW of the G-band results in an average value of $27 \pm 10 \mathrm{~cm}^{-1}$. This value is slightly higher those of $25 \pm 9 \mathrm{~cm}^{-1}$ previously determined for a hydrogen pre-treatment with subsequent graphene growth from a hydrogen/methane mixture on B2 copper ( $c f$. Figure 1f), thus proving the film to be of a lesser quality in this case when elemental hydrogen is not provided within gas atmosphere during the growth step. For B3 copper, the quality of the resulting Raman spectrum is even weaker (cf. SI Figure 4a,b). Figure $4 \mathrm{c}$ depicts the heating and gas phase protocol for an additional control experiment of a B2 copper substrate pre-treated in a carbon dioxide atmosphere prior to graphene growth from neat methane. The resulting film gives rise to a characteristic graphene-like Raman spectrum (Figure 4d), too. Compared to the previous hydrogen pre-treated sample ( $c f$. Fig. $4 \mathrm{~b}$ ), the intensity of the D band is reduced here (Figure $4 \mathrm{~d}$, left inset). The DRGW reveals an average of $22 \pm 10 \mathrm{~cm}^{-1}$, which is slightly higher than the value of $20 \pm 5 \mathrm{~cm}^{-1}$ extracted from the data of a sample obtained by carbon dioxide pre-treatment followed by an in-situ graphene growth in a hydrogen/methane mixture ( $c f$. Figure 1h). The DRGWs in Figure $4 \mathrm{~b}$ and Figure $4 \mathrm{~d}$ both exhibit a shoulder towards higher line width in the distribution curves, which can be explained by the build-up of disordered carbon while the graphene growth proceeds in the absence of hydrogen.

Therefore, considering the etching ability of carbon dioxide on carbon described before, it was fed in repeatedly into the growth stage in order to remove in-situ formed and co-deposited 
disordered carbon. This growth protocol is free of elemental hydrogen and consists of a pretreatment of the B2 copper substrate in a neat carbon dioxide atmosphere, followed by three repetitive treatments in a flow of neat methane for 10 minutes prior to the flow of a carbon dioxide / methane mix also applied for 10 minutes each ( $c f$. Figure 4e). The resulting sample exhibits a typical graphene Raman spectrum ( $c f$. Figure 4f) and the Raman D band becomes negligible (Figure 4f, left inset). By this pulsing procedure, also the DRGW distribution can be further improved since the shoulder towards larger line widths is no longer present and an average value of $18 \pm 8 \mathrm{~cm}^{-1}$ is obtained. This value is comparable to other literature ${ }^{12}$ and lower than the other DRGW values obtained in the present report. For B3 copper, similar results were achieved ( $c f$. SI Figure 4), indicating that the applied procedure leads to reproducible graphene film qualities irrespective of the substrate batches.

Noteworthy, the pulsing sequence results in a high quality graphene film, whereas a continuous growth experiment with an average gas flow of equal total amounts (i.e. $50 \mathrm{sccm}$ methane, 12.5 sccm carbon dioxide; $60 \mathrm{~min}, 1060^{\circ} \mathrm{C}$ in situ applied after the pre-cleaning with carbon dioxide) results in no graphene formation and carbon deposition. This indicates that the improved quality of the graphene film is rather obtained from the final step of the carbon dioxide pulsing sequence (cf. Figs. 4e,f). Thus, a reduction of the carbon dioxide content becomes evident. First results on further tuning the pulsed process into a continuous growth process lead to samples with high quality graphene on the copper substrate (e.g. by $50 \mathrm{sccm}$ methane, $3 \mathrm{sccm}$ carbon dioxide at the growth stage at $1060^{\circ} \mathrm{C}$ ). This is indicated by a defect free Raman spectrum of the as-grown sample on copper ( $c f$. SI Figure 6) with a G band width of approx. $16 \mathrm{~cm}^{-1}$, which is comparable to the line width of the G mode of a HOPG film measured under the same conditions as a reference. Whereas the CVD with neat methane leads to a film deposition with a high D-mode 
contribution ( $c f$. ref. 38 ), but the addition of a moderate carbon dioxide content to the methane gas phase results in a high quality graphene spectrum without detectable D- mode contribution (cf. Fig. SI 6), and an even higher carbon dioxide to methane ratio leads to no graphene and carbon deposition ( $c f$. previous discussion and Fig. 2c), the kinetic effect of a faster removal of defective carbon deposits compared graphene dissolution is obvious (there is no D- mode observed in Fig. SI 6). For a comparison, in Fig. SI 7 representative Raman spectra of a stronger carbon contaminated copper substrate are displayed after carbon dioxide pre-treatment.
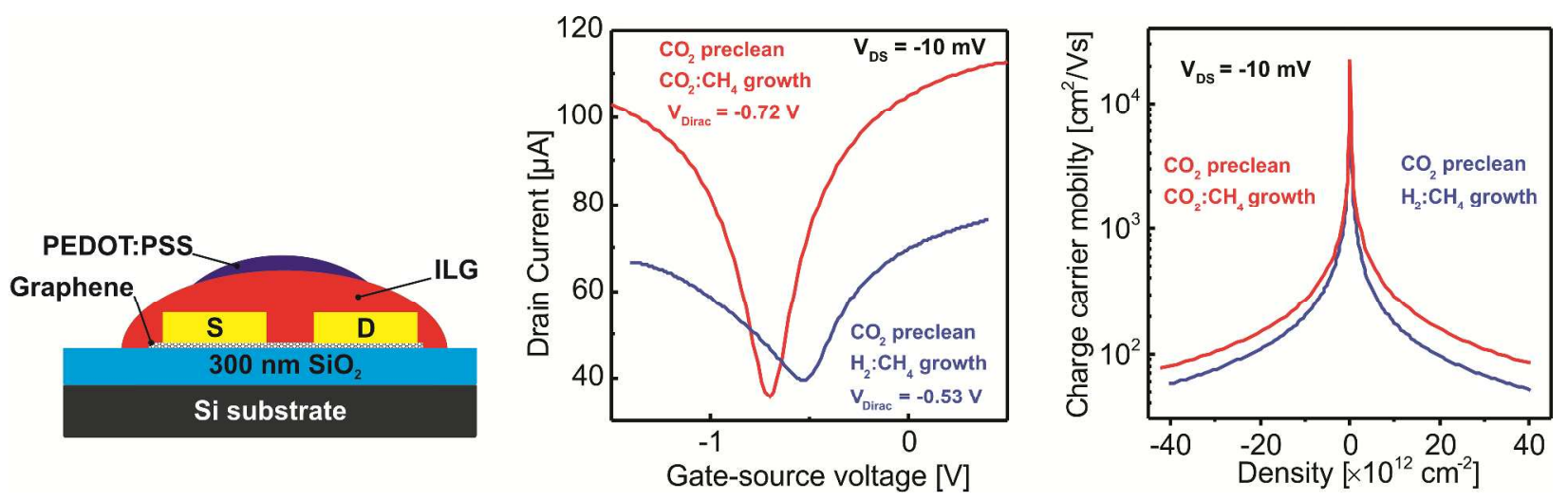

Figure 5: (a) Schematic cross section of the used setup for electrical characterization. (b) Transfer characteristics and (c) density dependence of the charge carrier mobility of graphene produced via two procedures. The first procedure (blue lines) involved a pre-cleaning stage in carbon dioxide followed by growth with hydrogen/methane ( $c f$. Figure 1e), the second procedure (red lines) involved a pre-cleaning stage in carbon dioxide followed by growth with carbon dioxide/methane ( $c f$. Figure 4e).

In order to characterize the influence of the modified protocols on the material properties of the graphene films we selected two graphene film samples grown on B2 copper for electrical characterization. The first sample underwent carbon dioxide annealing and hydrogen/methane 
growth (see heating protocol in Figure 2e) and the second sample was produced according to the procedure described in Figure 4e, essentially avoiding any hydrogen during the entire process. The graphene films were transferred by a standard poly(methyl methacrylate) (PMMA) assisted wet-transfer approach onto silicon wafers coated with silicon dioxide for the characterization of the electronic properties . Electrical measurements were performed in a field-effect transistor (FET) top-gate geometry (Figure 5a) with thermally evaporated gold source/drain contacts. The width $W$ of the transistor channel was $2000 \mu \mathrm{m}$, its length $L 100 \mu \mathrm{m}$. The ionic liquid gel (ILG) was based on a poly(vinylidene fluoride-hexafluoropropylene) polymer matrix and 1-ethyl-3methyl-imidazolium bis(trifluoromethylsulfonyl)imide as the ionic liquid. Figure 5b displays the transfer curves of FETs based on graphene grown from the two carbon dioxide assisted methods. The Dirac points in both samples are very close to each other $(-0.53 \mathrm{~V}$ for hydrogen/methane growth vs $-0.72 \mathrm{~V}$ for carbon dioxide/methane growth) indicating n-doping in both cases. The position of the Dirac point ${ }^{41}$ is similar to that observed in a previous work on IL-gated graphene in which mechanically exfoliated graphene was used. ${ }^{42}$

The charge carrier mobility $\mu$ has been calculated according to previously established protocols via the formula $\mu=(n e \rho)^{-1},{ }^{42}$ where $n$ is the charge carrier density, $e$ is the electron charge and $\rho$ the resistivity. The mobility is plotted as a function of doping density in Figure 5c. For our calculations, we have used a gate capacitance $C$ of $10 \mu \mathrm{F} / \mathrm{cm}^{2}$ that was derived from C-V measurements on one of the devices. Graphene produced with the carbon dioxide/methane protocol exhibits a larger $\mu$ than graphene grown with the standard hydrogen/methane growth mixture. Specifically, at a charge carrier density of $10^{12} \mathrm{~cm}^{-2}$, we calculate a mobility for the carbon dioxide/methane (hydrogen/methane) growth process of $1557 \mathrm{~cm}^{2} / \mathrm{Vs}\left(1379 \mathrm{~cm}^{2} / \mathrm{Vs}\right)$ for holes and $1975 \mathrm{~cm}^{2} / \mathrm{Vs}\left(1391 \mathrm{~cm}^{2} / \mathrm{Vs}\right)$ for electrons. Compared to electrochemically gated 
exfoliated graphene this value is three times larger than observed previously ${ }^{42}$ highlighting electrical quality of our graphene films. Our mobility values are comparable to the $2700 \mathrm{~cm}^{2} / \mathrm{Vs}$ described in CVD work elsewhere using back-gated devices and a graphene from standard hydrogen/methane based protocols ${ }^{43}$ The mobility values are however lower compared to the $5290 \mathrm{~cm}^{2} /$ Vs observed for CVD graphene from a hydrogen-free procedure in which the sample was transferred by a thermal release tape $\operatorname{method}^{8}$ and the $5100 \mathrm{~cm}^{2} / \mathrm{Vs}$ (effective Hall mobility) observed for CVD graphene transferred by roll-to-roll techniques. ${ }^{14}$

To further investigate the electrical properties over a larger scale, averaged four-point sheet resistance measurements were taken on both samples and yielded values of $0.92 \pm 0.07 \mathrm{k} \Omega / \square$ $(1.04 \pm 0.05 \mathrm{k} \Omega / \square)$ over the $2.5 \times 2.5 \mathrm{~cm}$ chip size for the carbon dioxide/methane (hydrogen/methane) based protocols, respectively. Again favorable results are observed from the carbon dioxide/methane based protocol indicating the good quality of the graphene. The sheet resistance values for both protocols are higher than those quoted elsewhere $(0.2-0.3 \mathrm{k} \Omega / \square)$ for both hydrogen-containing ${ }^{14}$ and hydrogen-free ${ }^{8}$ processes. This can be explained by the low level of doping observed within the films of this study and by the impact of the transfer protocols on the doping. The convergence of the sheet resistance values when measured on millimeter scales is likely due to the presence of defects on the rims of mesoscale graphene domains and defects induced during the transfer stage. ${ }^{44}$

\section{Conclusion}

The use of carbon dioxide in the synthesis of graphene by CVD enables a uniform preconditioning of differently behaving copper substrates of comparable quality and results in the formation of high quality films. We have found that graphene can be grown even on heavily 
"carbon-contaminated" copper foils from various batches by pre-treatment of the copper substrates in a carbon dioxide atmosphere and developed a growth protocol on carbondioxide/methane only, free of elemental hydrogen for graphene films. Average Raman G band widths as low as $18 \mathrm{~cm}^{-1}$ could be extracted from DRGW plots and the half width of all constituting 2D band features is of approx. $31 \mathrm{~cm}^{-1}$. Electron charge carrier mobilities of up to $1975 \mathrm{~cm}^{2} / \mathrm{Vs}$ were observed for transferred films on $\mathrm{Si} / \mathrm{SiO}_{2}$. We could demonstrate how a carbon dioxide based CVD growth chemistry allows the removal of multiple carbon impurities from copper substrates commonly used in the field and enables a consistent, scalable and uniform growth of high quality graphene films irrespective of the substrate nature. A comparable effect could not be observed by thermal hydrogen pre-treatment which is currently the used in most reports on CVD graphene production. A failure to remove carbon impurities prior to the growth step is proven to seriously inhibit the subsequent CVD synthesis of high quality graphene films and results in an incomplete coverage and the presence of disordered carbon on the surface. These limiting factors are addressable by the CVD parameters and influence the individual domain sizes and grain boundaries, sheet resistance and carrier mobility of the graphene films, whereas the latter two are affected by subsequent wet chemical transfer techniques, too.

The further investigation of the parameter range of scalable and less hazardous carbon dioxide based, pulsed or continuous growth protocols will allow to understand the selective etching of less ordered $\mathrm{sp}^{2}$ - carbon phases and non-graphene type carbon by carbon dioxide in more detail, and the screening and correlation of the transfer methods with the electrical properties are considered key next steps towards the reproducible production of high quality graphene films on target substrates for devices and commercial applications. 


\section{Methods}

All copper foils were purchased from Alfa Aesar and had a common $0.025 \mathrm{~mm}(0.001$ in) thickness and 99.8\% (metals basis) purity. Batch 1 (B1), product no. 46986, annealed, uncoated. Batch 2 (B2), product no. 13382, annealed, coated. Batch 3 (B3), product no. 46365, annealed, uncoated. For reference in SI Figure 1c, a high purity copper substrate was screened, too (Alfa Aesar product no. 42972, 99.9999\% purity, $0.05 \mathrm{~mm}$ ).

For CVD experiments B1, B2 and B3 copper foils were heated for times at temperatures and under gas compositions as described in the text. Foils were cleaned two times with dichloromethane (Sigma Aldrich, Product number: 270997, anhydrous, $\geq 99.8 \%$, contains 50$150 \mathrm{ppm}$ amylene as stabilizer), in two separate baths. The foils were then allowed to dry in air before being loaded into a quartz glass tube furnace. The substrates were suspended on quartz glass holders in the gas flow and the system leak rate was maintained below a value of 0.25 $\mathrm{mbar} /$ hour prior to the experiments. A heating rate of $20 \mathrm{~K} / \mathrm{min}$ was applied for reaching the top temperature and accurate temperature readings were made by using thermocouples situated on the outside and the inside the tube furnace. The maximum discrepancy between the outside and internal thermocouples was approximately $+18{ }^{\circ} \mathrm{C}$ (according to an internal temperature of 1078 $\left.{ }^{\circ} \mathrm{C}\right)$ at the growth temperature of $1060{ }^{\circ} \mathrm{C}(2 \%$ difference $)$. The gas flow during the processes was controlled by mass flow controllers with a tolerance of $2 \%$. The pressure values were measured using Pirani gauges (Pfeiffer Vacuum, TPR 281) and verified to be within $10 \%$ accuracy via the use of capacitive gauges (Pfeiffer Vacuum, CMR 361,365) in parallel [system base pressure: $2 \times 10^{-3}$ mbar; representative Pirani- and capacive sensors pressure readings for 150 sccm $\mathrm{H}_{2}: 0.44 / 0.31 ; 150 \mathrm{sccm} \mathrm{H} \mathrm{H}_{2}+50 \mathrm{sccm} \mathrm{CH}$ : 0.74/0.54; $50 \mathrm{sccm} \mathrm{CO}_{2}: 0.16 / 0.15 ; 50 \mathrm{sccm}$ $\left.\left.\mathrm{CH}_{4}+3 \mathrm{sccm} \mathrm{CO} 20.22 / 0.14\right)\right]$ 
Raman mapping was carried using a NT-MDT NTEGRA spectrometer using a $100 \mathrm{X}$ optical objective with an average spot size of around $1 \mu \mathrm{m}$. A laser excitation wavelength of $514 \mathrm{~nm}$ was used, the diffraction grating used had 600 lines/mm and resulting in a spectral resolution of approximately $1 \mathrm{~cm}^{-1}$. During all measurements there was no observation of the Raman laser altering the sample composition or causing sample burning (observed by monitoring the spectra from a single point repeatedly over time during the focusing process). Fits to the $\mathrm{G}$ band were carried out using a standard Lorentzian line shape. Typically three Raman maps of 20 X 20 data points over a $20 \mu \mathrm{m}$ X $20 \mu \mathrm{m}$ area were collected from each sample and the G band width from all these scans was grouped into histogram bins and the mean value $(\mu)$ and standard deviation $(\sigma)$ extracted from a normal distribution fit to this data. Data from spectra where the fit process fails are filtered from the histograms and mean value calculations.

Scanning electron microscopy (SEM) was performed with a Zeiss LEO 1530 and energy dispersive X-ray spectroscopy (EDX) was performed with a Gemini at $1.0 \mathrm{keV}$ and Hitachi SU8000 at $1.0 \mathrm{keV}$.

For the electrical measurements, graphene was transferred to $\mathrm{Si} / \mathrm{SiO}_{2}$ wafers $\left(300 \mathrm{~nm}\right.$ of $\left.\mathrm{SiO}_{2}\right)$ via a wet transfer method using a spin coated (2000 RPM, 1 min) poly(methyl methacrylate) layer (PMMA, molecular weight 996000, $10 \mathrm{wt} \%$ solution in toluene) to add structural stability to the graphene film. Ammonium peroxodisulfate solution (3 g per $100 \mathrm{ml}$ deionized water) was used to etch off the copper substrate. The PMMA-graphene stack was then rinsed three times with deionized water to clear excess etching solution before transfer to the $\mathrm{Si} / \mathrm{SiO}_{2}$ substrate. The PMMA layer was then removed using an acetone bath.

The electrical measurements were performed in a top contact top gate geometry (Figure 5a) using thermally evaporated gold contacts with a $W / L$ ratio of 20 as source/drain contacts and an 
ionic liquid gel (ILG) based on a poly(vinylidene fluoride-hexafluoropropylene) matrix and 1ethyl-3-methyl-imidazolium bis(trifluoromethylsulfonyl)imide room-temperature ionic liquid as gate dielectric. A drop of poly(3,4-ethylenedioxythiophene) polystyrene sulfonate (PEDOT:PSS) was placed on top of the ILG to allow for easier contacting of the ILG via probe needles. All measurements were performed in ambient air.

For four-point sheet resistance measurements, the same transfer process was used. The samples were contacted with a linear four-pint Jandel probe (1 mm needle spacing) and a Keithley 2400 Sourcemeter was used to obtain resistance values on 10 points covering the sample area. The average of these values $(R)$ was then converted to a sheet resistance $\left(R_{\square}\right)$ using:

$$
R_{\square}=\frac{\pi}{\ln 2} R
$$

\section{Supporting Information}

Supporting Information is available free of charge via the Internet at http://pubs.acs.org.

\section{Acknowledgment}

This work was financially supported by a Marie-Curie Fellowship within the frame of the GENIUS project (FP7 - PEOPLE Programme) and the ERC Advanced Grant NANOGRAPH. The authors would like to thank G. Glaßer (MPI-P Mainz) for SEM and EDX measurements and Benjamin Rietz (BASF SE) for assistance in CMIC. 


\section{REFERENCES}

[1] Mattevi, C.; Kim, H.; Chowalla, M. A Review of Chemical Vapor Deposition of Graphene on Copper. $J$. Mater. Chem. 2011, 21, 3324-3334.

[2] Novoselov, K. S.; Fal'ko, V. I.; Colombo, L.; Gellert, P. R.; Schwab, M. G.; Kim, K. A Roadmap for Graphene. Nature. 2012, 490, 192-200.

[3] Yu, Q.; Lian, J.; Siriponglert, S.; Li, H.; Chen, Y. P.; Pei, S. -S. Graphene Segregated on Ni Surfaces and Transferred to Insulators. Appl. Phys. Lett. 2008, 93, 113103.

[4] Wei, D.; Lie, Y.; Wang, Y.; Zhang, H.; Huang, L.; Yu, G. Synthesis of N-Doped Graphene by Chemical Vapor Deposition and its Electrical Properties. Nano Lett. 2009, 9, 1752-1758.

[5] Lee, Y.; Bae, S.; Jang, S.; Zhu, S. -E.; Sim, S. H.; Song, Y. I.; Hong, B. H.; Ahn, J. -H. Wafer-scale Synthesis and Transfer of Graphene Films. Nano Lett. 2010, 10, 490-493.

[6] Yuan, W.; Shi, G. Graphene-Based Gas Sensors. J. Mater. Chem. A. 2013, 1, 10078-10091.

[7] Park, S.; An, J.; Suk, J. W.; Ruoff, R. S. Graphene-Based Actuators. Small. 2010, 6, 210-212.

[8] Ryu, J.; Kim, Y.; Won, D.; Kim, N.; Park, J. S.; Lee, E. -K.; Cho, D.; Cho, S. -P.;Kim, S. J.; Ryu, G. H. et al. Fast Synthesis of High-Performance Graphene Films by Hydrogen-Free Rapid Thermal Chemical Vapor Deposition. ACS Nano. 2013, 8, 950-956.

[9] Sutter, P. W.; Flege. J.-I.; Sutter, E. A. Epitaxial Graphene on Ruthenium. Nat. Mater. 2008, 7, 406-411.

[10] Reina, A.; Son, H.; Jiao, L.; Fan, B.; Dresselhaus, M. S.; Liu, Z.; Kong, J. Transferring and Identification of Single-and Few-Layer Graphene on Arbitrary Substrates. J. Phys. Chem. C. 2008, 112, 17741-17744.

[11] Li, X.; Cai, W.; An, J.; Kim, S.; Nah, J.; Yang, D.; Piner, R.; Velamakanni, A.; Jung, I.; Tutuc, E. et al . Large-Area Synthesis of High-Quality and Uniform Graphene Films on Copper Foils. Science. 2009, 324, 13121314.

[12] Kidambi, P. R.; Ducati, C.; Dlubak, B.; Gardiner, D.; Weatherup, R. S.; Martin, M. -B.; Seneor, P.; Coles, H.; Hofmann, S. The Parameter Space of Graphene Chemical Vapor Deposition on Polycrystalline Cu. J. Phys. Chem. C. 2012, 116, 22492-22501.

[13] Kim, K. S.; Zhao, Y.; Jang, H.; Lee, S. Y.; Kim, J. M.; Kim, K. S.; Ahn, J, -H.; Kim, P.; Choi, J. -Y.; Hong, B. H. Large-Scale Pattern Growth of Graphene Films for Stretchable Transparent Electrodes. Nature. 2009, 457, 706-710.

[14] Bae, S.; Kim, H.; Lee, Y.; Xu, X.; Park, J. S.; Zheng, Y.; Balakrishnan, J.; Lei, T.; Kim, H. R.; Song, Y. I. et al. Roll-to-Roll Production of 30-inch Graphene Films for Transparent Electrodes. Nat. Nanotechnol. 2010, 5, 574578.

[15] Gao, L.; Ni, G. -X.; Liu, B.; Castro Neto, A. H.; Loh, K. P. Face-to-face Transfer of Wafer-scale Graphene Films. Nature. 2014, 190-194.

[16] Kobayashi, T.; Bando, M.; Kimura, N.; Shimizu, K.; Kadono, K. Production of a 100-m-long High-Quality Graphene Transparent Conductive Film by Roll-to-Roll Chemical Vapor Deposition and Transfer Process. Appl. Phys. Lett. 2013, 102, 023112.

[17] Wu, Y. A.; Fan, Y.; Speller, S.; Creeth, G. L.; Sadowski, J. T.; He, K.; Robertson, A. W.; Allen, C. S.; Warner, J. H. Large Single Crystals of Graphene on Melted Copper Using Chemical Vapor Deposition. ACS Nano. 2012, 6, 5010-5017. 
[18] Geng, D.; Wu, B.; Guo, Y.; Huang, L.; Xue, Y.; Chen, J.; Yu, G.; Jiang, L.; Hu, W.; Liu, Y. Uniform Hexagon Graphene Flakes and Films Grown on Liquid Copper Surface. Proc. Natl. Acad. Sci. U.S.A. 2012, 109, 7992-7996.

[19] Ago, H.; Ogawa, Y.; Tsuji, M.; Mizuno, S.; Hibino, H. Catalytic growth of graphene: Toward large-area single-crystalline graphene. J. Phys. Chem. Lett. 2012, 3, 2228-2236.

[20] Kato, R.; Tsugawa, K.; Okigawa, Y.; Ishihara, M.; Yamada, T.; Hasegawa, M.; Bilayer graphene synthesis by plasma treatment of copper foils without using a carbon containing gas. Carbon 2014, 77,823-828.

[21] Wan, X.; Chen, K.; Xu, J.; Interface Engineering for CVD Graphene: Current Status and Progress. Small 2014, DOI: $10.1002 /$ smll.201401458

[22] Kobayashi, T.; Hirakuri, K. K.; Mutsukura, N.; Machi, Y. Synthesis of CVD Diamond at Atmospheric Pressure Using the Hot-Filament CVD Method. Diamond Relat. Mater. 1999, 8, 1057-1060.

[23] Nakahigashi, T.; Tanaka, Y.; Miyake, K.; Oohara, H. Properties of Flexible DLC Film Deposited by Amplitude-Modulated RF P-CVD. Tribol. Int. 2004, 37, 907-912.

[24] Hao, Y.; Bharathi, M. S.; Wang, L.; Liu, Y.; Chen, H.; Nie, S.; Wang, X.; Chou, H.; Tan, C.; Fallahazad, B. et al. The Role of Surface Oxygen in the Growth of Large Single-Crystal Graphene on Copper. Science. 2013, 342, 720-723.

[25] Magnuson, C. W.; Kong, X. Copper Oxide as a "Self-Cleaning" Substrate for Graphene Growth. J. Mater. Res. 2014, 29, 403-409.

[26] Kato, R.; Tsugawa, K.; Okigawa, Y.; Ishihara, M.; Yamada, T.; Hasegawa, M. Bilayer Graphene Synthesis by Plasma Treatment of Copper Foils Without Using a Carbon-containing Gas. Carbon. 2014, 77, 823-828.

[27] Elias, D. C.; Nair, R. R.; Mohiuddin, T. M. G.; Morozov, S. V.; Blake, P.; Halsall, M. P.; Ferrari, A. C.; Boukhvalov, D. W.; Katnelson, M. I.; Geim, A. K.; Novoselov, K. S. Control of Graphene's Properties by Reversible Hydrogenation: Evidence for Graphane. Science. 2009, 323, 610-613

[28] Haberer, D.; Giusca, C. E.; Wang, Y.; Sachdev, H.; Fedorov; A. V.; Farjam, M.; Jafari, A.; Vyalikh, D. V.; Usachov, D.; Liu, X. et al. Adv. Mater. 2011, 23, 4497-4503.

[29] Ferrari, A. C.; Meyer, J. C.; Scardaci, V.; Casiraghi, C.; Lazzeri, M.; Mauri, F.; Pisanec, S.; Jiang, D.; Novoselov, K. S.; Roth, S.; Geim, A. K. Raman Spectrum of Graphene and Graphene Layers. Phys. Rev. Lett. 2006, 97, 187401.

[30] Ferrari, A. C. Raman Spectroscopy of Graphene and Graphite: Disorder, Electron-Phonon Coupling, Doping and Nonadiabatic effects. Solid State Commun. 2007, 143, 47-57.

[31] Ferrari, A. C.; Basko, D. M. Raman Spectroscopy as a Versatile Tool for Studying the Properties of Graphene. Nat. Nanotechnol. 2013, 8, 235-246.

[32] López, G. A.; Mittemeijer, E. J. The Solubility of C in Solid Cu. Scr. Mater. 2004, 51, 1-5.

[33] A. Holleman, E. Wiberg; ed. N. Wiberg: Lehrbuch der Anorganischen Chemie, 102. Aufl. 2007, W. de Gruyter, Berlin

[34] Sachdev, H. Diamonds From the Pressure Cooker - Science or Science Fiction? Angew. Chem. 2004, 116, 4800-4803

[35] Monachino, E.; Greiner, M.; Knop-Gericke, A.; Schloegl, R.; Dri, C.; Vesselli, E.; Comelli, G.; Reactivity of Carbon Dioxide on Nickel: Role of CO in the Competing Interplay between Oxygen and Graphene. J. Phys. Chem. Lett. 2014, 5, 1929-1934. 
[36] Kim, S. M.; Hsu, A.; Lee, Y. H.; Dresselhaus, M.; Palacios, T.; Kim, K. K.; Kong, J. The Effect of Copper Pre-cleaning on Graphene Synthesis. Nanotechnology. 2013, 24, 365602.

[37] Losurdo, M.; Giangregorio, M. M.; Capezzuto, P.; Bruno, G. Graphene CVD Growth on Copper and Nickel: Role of Hydrogen in Kinetics and Structure. Phys. Chem. Chem. Phys. 2011, 13, 20836-20843.

[38] Ito, Y.; Christodoulou C.; Nardi, M. V.; Koch, N.; Sachdev, H.; Müllen, K. Chemical Vapor Deposition of N-doped Graphene and Carbon Films: The Role of Precursors and Gas Phase. ACS Nano. 2014, 8; 3337 - 3346.

[39] Bernard, L. S.; Spina, M.; Jacimovic, J.; Ribic, P. R.; Walter, A.; Oberli, D. Y.; Horvath, E.; Forro, L.; Magrez, A. Functionalized Graphene Grown by Oxidative Dehydrogenation Chemistry. Carbon. 2013, 71, 11-19.

[40] Müller, F.; Sachdev, H.; Hüfner, S.; Pollard, A. J.; Perkins, E. W.; Russell, J. C.; Beton, P. H.; Gsell, S.; Fischer, M.; Schreck, M.; Stritzker, B. How Does Graphene Grow? Easy Access to Well-Ordered Graphene Films. Small. 2009, 5, 2291-2296.

[41] Castro Neto, A. H.; Guinea, F.; Peres, N. M. R.; Novoselov, K. S.; Geim, A. K. The Electronic Properties of Graphene. Rev. Mod. Phys. 2009, 81, 109-162.

[42] Das, A.; Pisana, S.; Chakraborty, B.; Pisanec, S.; Saha, S. K.; Waghmare, U. V.; Novoselov, K. S.; Krishnamurthy, H. R.; Geim, A. K.; Ferrari, A. C.; Sood, A. K. Monitoring Dopants by Raman Scattering in an Electrochemically Top-Gated Graphene Transistor. Nat. Nanotechnol. 2008, 3, 210-215.

[43] Pirkle, A.; Chan, J.; Venugopal, A.; Hinojos, D.; Magnuson, C. W.; McDonnell, S.; Colombo, L.; Vogel, E. M.; Ruoff, R. S.; Wallace, R. M. The Effect of Chemical Residues on the Physical and Electrical Properties of Chemical Vapor Deposited Graphene Transferred to $\mathrm{SiO}_{2}$. Appl. Phys. Lett. 2011, 99, 122108

[44] Kang, J.; Shin, D.; Bae, S.; Hong, B. H. Graphene Transfer: Key for Applications. Nanoscale. 2012, 4, 55275537 . 
ToC Graphical Abstract $254 \times 190 \mathrm{~mm}(72 \times 72 \mathrm{DPI})$ 
c) carbon dioxide pre-treatment without subsequent CVD graphene growth

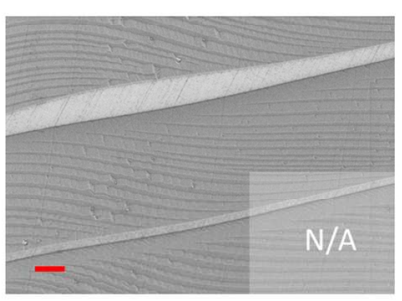

hydrogen pre-treatment with subsequent CVD graphene growth

e)

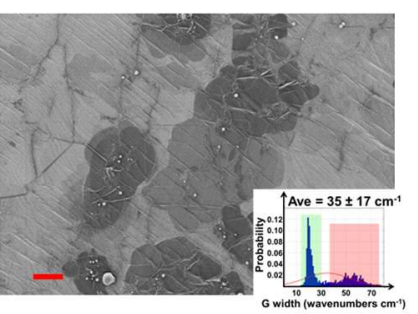

carbon dioxide pre-treatment with subsequent CVD graphene growth

g)

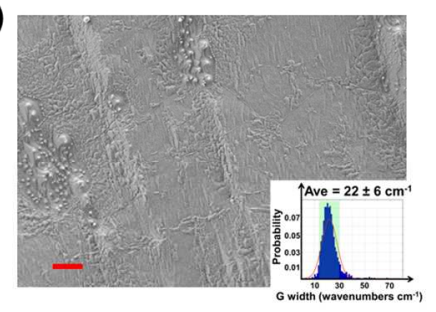

d)
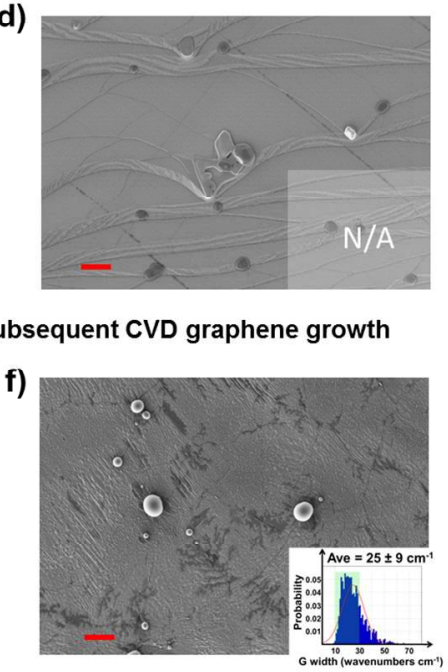

Batch 2

b)

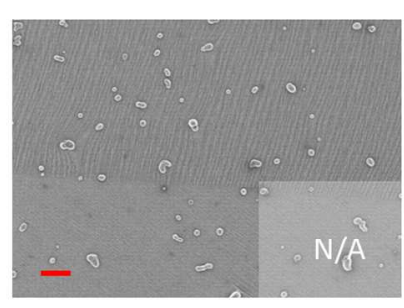

h)

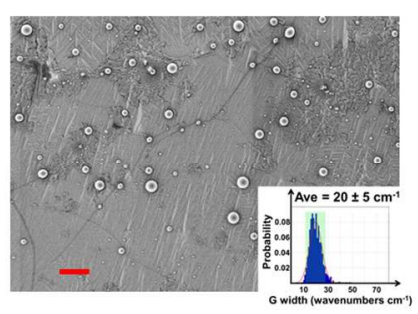

Figure 1 $205 \times 305 \mathrm{~mm}(150 \times 150 \mathrm{DPI})$ 
Qapge 33 of 36 Sample $X$

b)

SAarpienX $+\mathrm{H}_{2}$

c)

Sample $\mathrm{X}+\mathrm{CO}_{2}$
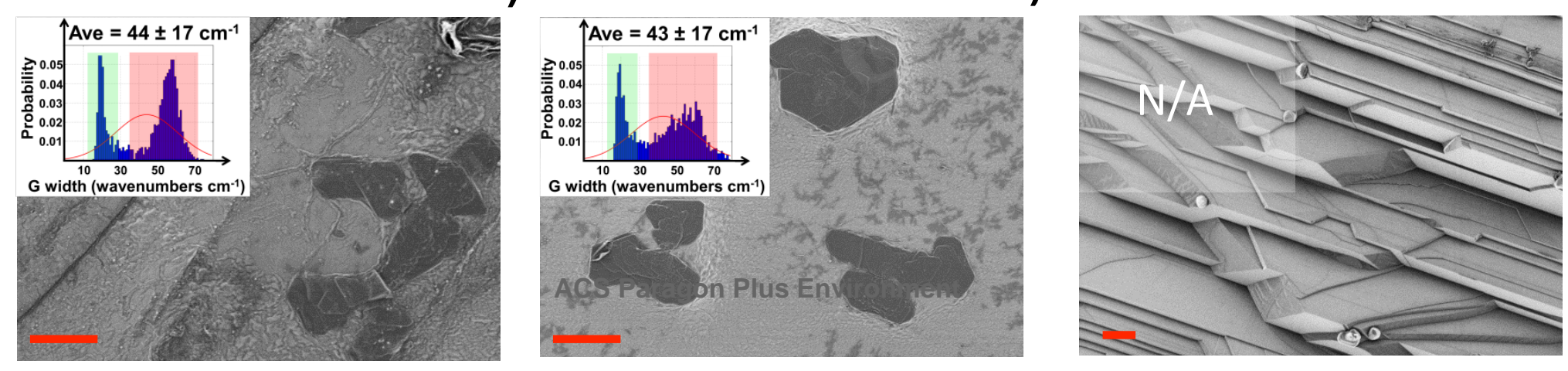


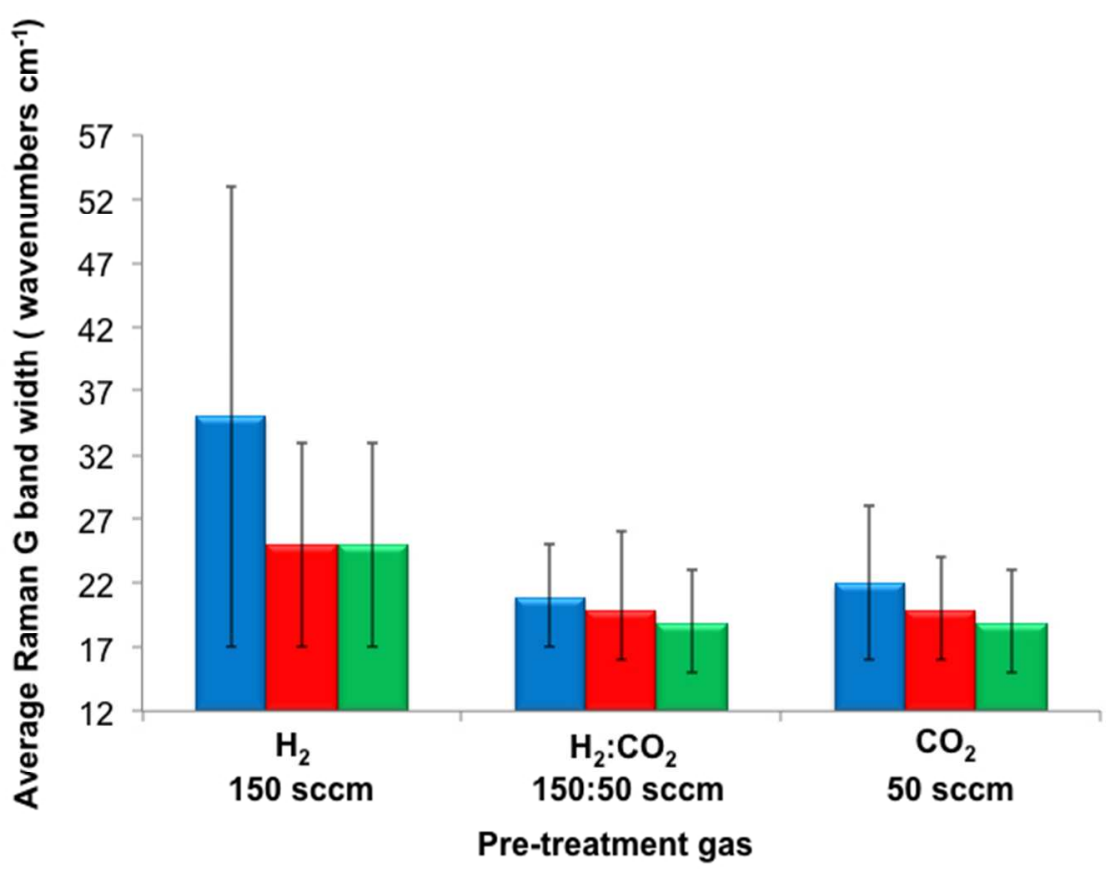

Figure 3

$254 \times 190 \mathrm{~mm}(72 \times 72 \mathrm{DPI})$ 


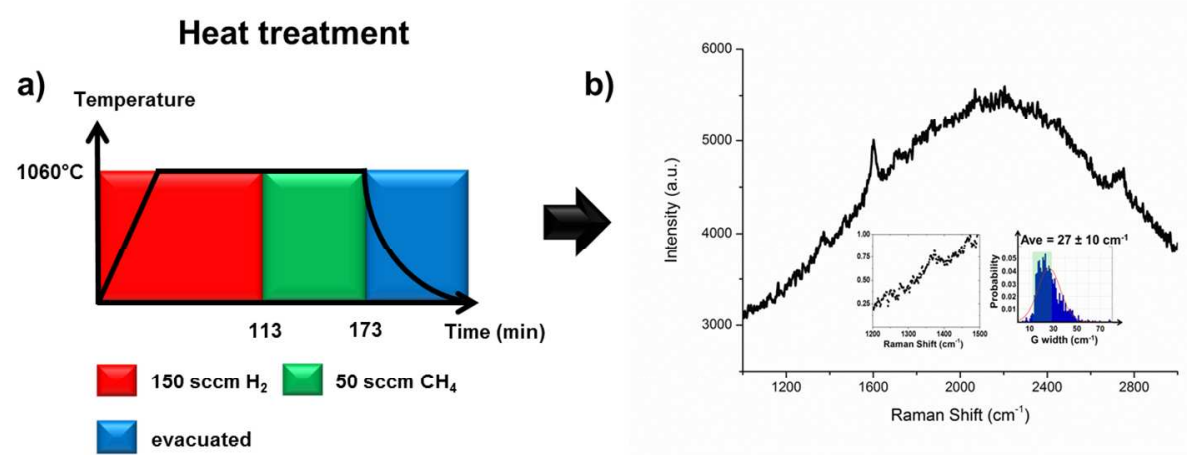

c)
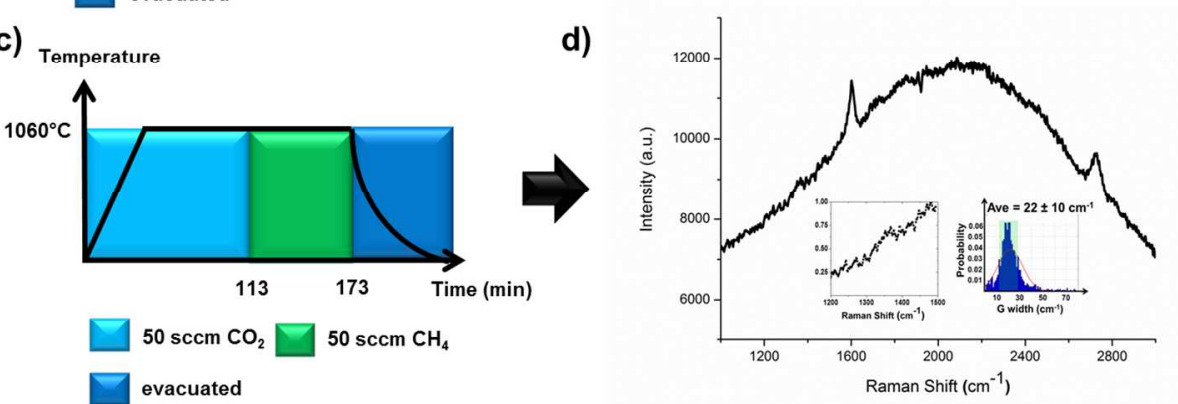

e)
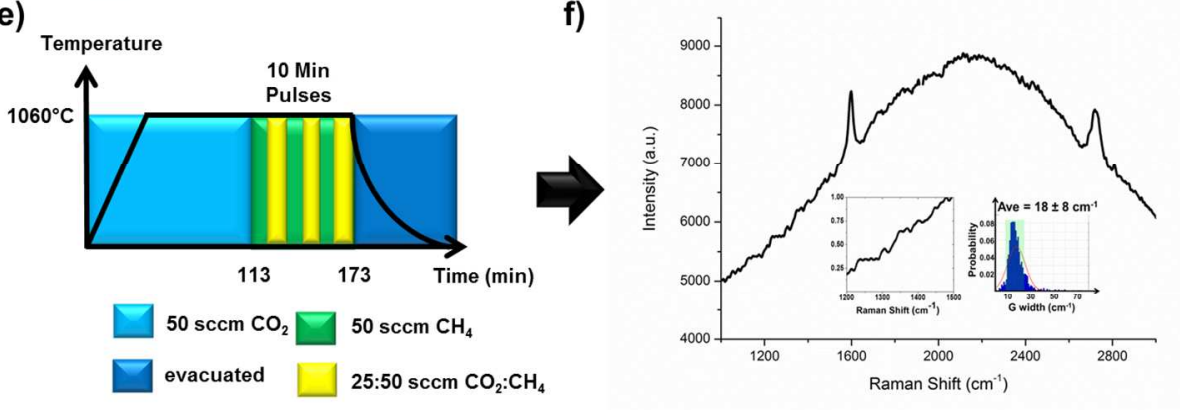

Figure 4 $252 \times 251 \mathrm{~mm}(150 \times 150 \mathrm{DPI})$ 

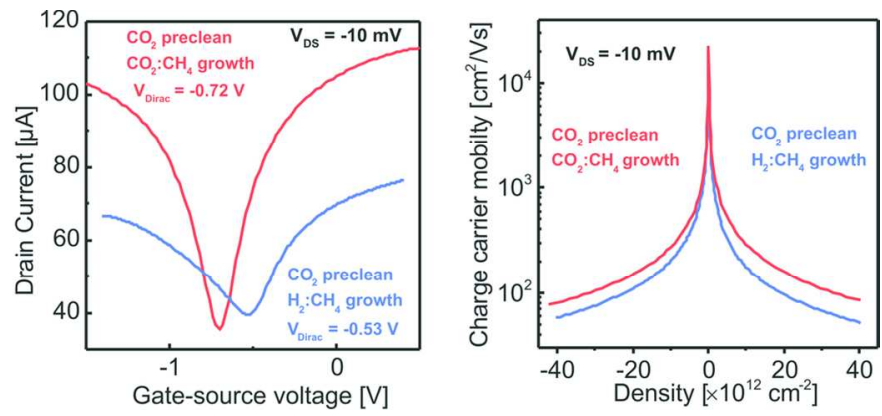

Figure 5

$125 \times 38 \mathrm{~mm}(300 \times 300$ DPI $)$ 